\title{
Design and dynamic characterization of an orientation insensitive microwave water-cut sensor
}

\author{
Muhammad Akram Karimi ${ }^{1}$, Student Member, IEEE, Muhammad Arsalan ${ }^{2}$, and Atif Shamim ${ }^{1}$, Senior \\ Member, IEEE
}

\begin{abstract}
Modern reservoir management in oil and gas industry relies on accurate water fraction measurement which is produced as a by-product with oil. This paper presents a novel and contactless water fraction (also known as water cut) measurement technique which is independent of geometric distribution of oil and water inside the pipe. The sensor is based on a modified T-resonator implemented directly on the pipe's outer surface and whose resonance frequency decreases by increasing the water content in oil. The E-fields have been made to rotate and distribute well inside the pipe, despite having narrow and curved ground plane. It makes the sensor's reading dependent only on the water fraction and not on the mixture distribution inside the pipe. That is why, the presented design does not require any flow conditioner to homogenize the oil/water mixture unlike many commercial WC sensors. The presented sensor has been realized by using extremely low cost methods of screen printing and reusable 3D printed mask. Complete characterization of the proposed WC sensor, both in horizontal and vertical orientations, has been carried out in an industrial flow loop. Excellent repeatability of the sensor's response has been observed in "dispersed bubble" as well as in "stratified wavy" flow regimes. The performance test of the sensor confirms that the water fraction measurement is independent of the flow pattern, flow rate or orientation. The measured performance results of the sensor show full range accuracy of $\pm 2-3 \%$ while tested under random orientations and wide range of flow rates.
\end{abstract}

Index Terms-3D printing, flow loop, flow regime, microwave sensor, reservoir management, screen printing, $T$ resonator, water-cut

\section{INTRODUCTION}

$\mathrm{E}$ FFECTIVE oil production is indispensable in order to fulfill the monotonically increasing energy demands of the world. Modern reservoir management requires real time monitoring of water fraction that is produced as a by-product with oil. Its amount varies with the age and geolocation of the oil well which requires the water-cut (WC) sensors to cover full range. With the proliferation of multilateral oil wells, the

Manuscript received on October 31, 2016

1. Authors are with Integrated Microwave Packaging Antennas and Circuits Technology (IMPACT), at the Computer, Electrical, and Mathematical Sciences and Engineering Division, King Abdullah University of Science and Technology, 4700 KAUST, 23955 Thuwal, Kingdom of Saudi Arabia (email: muhammadakram.karimi@kaust.edu.sa)

2. Author is with EXPEC ARC, Production Technology Team in Saudi Aramco, Kingdom of Saudi Arabia (muhammad.arsalan@aramco.com) need of low cost water-cut (WC) sensing ${ }^{1}$ nodes that can be used in both horizontal as well as vertical installations has increased [1]. Real time monitoring through inline WC sensors installed at all the branches of a multi-lateral oil well enables not only proactive (versus reactive) reservoir management but also helps in efficient production allocation and flow assurance [2]. Water produced The WC sensors available at the moment are either costly, heavy, intrusive, incapable of covering full WC range and/or require mixer as flow conditioner.

Inline detection of water fraction in oil can be done by sensing the differences in the density or effective electric permittivity or IR/gamma ray absorption characteristics of oil/water (O/W) mixture. Recently, Aramco has developed a WC sensor based upon the difference in densities of oil and water which affects the speed of sound (SoS) travelling through it [1]. The flow loop testing of the sensor shows an accuracy of $\pm 5 \%$ provided that the sensor is installed near $90^{\circ}$ bend to help homogenization of the $\mathrm{O} / \mathrm{W}$ mixture. A homogenizer (mixer) has been recommended for accurate results. Similarly, a WC meter produced commercially by ESSIFLO is dielectric constant based sensor which comes with inline static mixer helping homogenizing the $\mathrm{O} / \mathrm{W}$ mixture [3]. The reliance on the use of such mixers cause undesirable pressure drop in the pipelines which increases the overall cost of oil production.

Most of the commercially available WC sensors, like the one from Delta-C, are based upon measuring the capacitance of $\mathrm{O} / \mathrm{W}$ mixture which can cover limited water cut range (typically from $0 \%$ to $70-80 \%$ ) only [4], [5]. Capacitive sensing can be broadly categorized based on the excitation frequency of measurements. The low excitation frequency (in the range of $\mathrm{kHz}$ ) measurement method is known to suffer from a short circuit effect between the capacitor electrodes in the presence of a conductive medium (i.e., in the case of high WCs when water is the continuous phase); therefore, this method is not applicable for reliable WC measurements beyond a WC of $70 \%$ [6].

Some of the commercially available water-cut sensors, as the one from Phase Dynamics, are based on coaxial microwave resonators whose central rod disturbs the smooth

\footnotetext{
${ }^{1} \mathrm{WC}$ sensing is to sense the volumetric fraction of water in oil
} 
flow of the O/W mixture in the pipeline [7], [8]. The WC sensor by Roxar is based

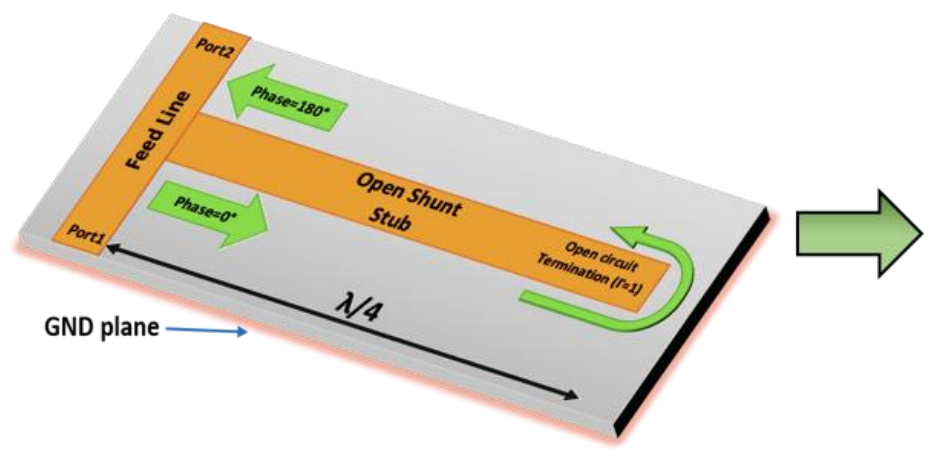

(a)

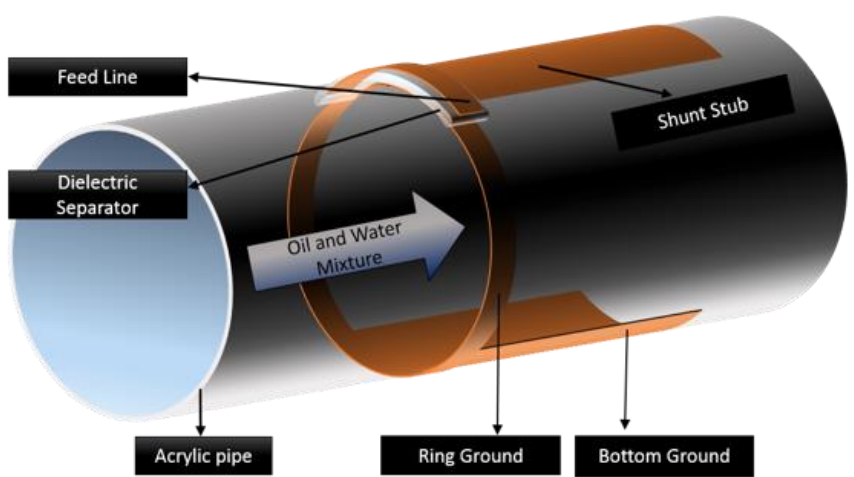

(b)

Fig. 1. Conventional T-resonator on a flat substrate (b) Implementation of a T-resonator on cylindrical pipe surface using a modified ground plane

on microwave cylindrical fin resonator (CFR) which also hinders the flow of mixture [9]. Microwave transmission based WC sensors are limited to smaller pipe sizes as the loss increases proportional to the diameter of the pipe [10] [11]. Similarly the WC sensors based on IR spectroscopy, like the one from Weatherford, performs its spectroscopy on the small portion of the mixture and also intrudes the mixture flowing through the pipeline [12]. "Vx Spectra" developed by Schlumberger makes use of multi-energy gamma rays which can measure the phase fractions of oil, water and gas [13], [14]. As the gamma rays make use of radioactive sources, its handling and disposal becomes a critical safety issue.

Our earlier work presents a microwave, pipe conformable WC sensor which was tested in static conditions [15]. The earlier design works well as long as oil and water are well mixed or do not change their geometric distribution inside the pipe. In real scenario, oil and water are randomly mixed, generating complex flow regimes. Like most of the commercial WC sensors, our earlier design can work well only if a mixer is installed before the sensor. In order to solve this issue, this paper presents a new design that is extremely low cost, almost weightless, completely nonintrusive, covers full range and is orientation insensitive as well. Unlike many commercial WC sensors, the presented sensor does not even require any mixer as a flow conditioner. The sensor has been fabricated using low cost methods of manual screen printing of silver paste patterned using a reusable $3 \mathrm{D}$ printed mask. The performance of the fabricated prototype has been tested in an industrial flow loop where the sensor worked equally well in horizontal as well as in vertical installations despite having completely different flow regimes, i.e. wavy stratified and dispersed bubble, respectively. The sensor showed an accuracy within $\pm 2-3 \%$ over full range (0-100\%) despite having an uncertainty of $\pm 2.5 \%$ in setting the $\mathrm{WC}$ in the flow loop

\section{DESIGN}

The designed WC sensor is based upon the principle of T-resonance which is used to measure the dielectric properties of the substrates [16]. A typical microstrip based T-resonator is a 2 port single ended network consisting of a feedline and a $\lambda / 4$ open circuit shunt stub. At resonance frequency, the shunt stub appears as a short circuit (after $\lambda / 4$ transformation) and inhibits the signal propagation from port 1 of the feedline to port 2, as shown in Fig. 1 (a). That is why, the resonance condition is characterized by a dip in the transmission coefficient $\left(\mathrm{S}_{21}\right)$ whose frequency depends on the dielectric properties of the substrate.

Although microfluidic sensors have been built to find the dielectric properties of different mixtures [17], the main challenge here was to implement the T-resonator directly on the pipe surface to achieve non-intrusiveness and low cost realization. Our earlier work [15] explains the basic implementation of T-resonator on the pipe surface by using modified ground plane which was then used to characterize different fractions of water in oil statically. A representative diagram of the design is shown in Fig. 1 (b). The extension of the bottom ground plane in the form of a ring helps in the matching of the feedline with $50 \Omega$ even in the presence of different substrates inside the pipe. It can be seen from the figure that the shunt stub and bottom ground plane lie flat on the pipe surface so we will call this design as "Flat design" from now onwards.

\section{A. Drawback in Flat Design}

The response of an ideal WC sensor should depend only on the relative percentage of oil/water and no on the geometric distribution inside the pipe. In order to see how the flat sensor performs in different geometric distributions of oil and water; we simulated it in HFSS. As we know that the E-field distribution inside the pipe determines the response of the sensor so we have plotted the fields in the pipe cross section as shown in Fig. 2. Change in magnitude of $\mathrm{E}$ fields inside the pipe is due to fact that the pipe thickness is comparable to the wavelength at frequency of 
operation $(100-200 \mathrm{MHz})$ and the bottom ground size is limited.

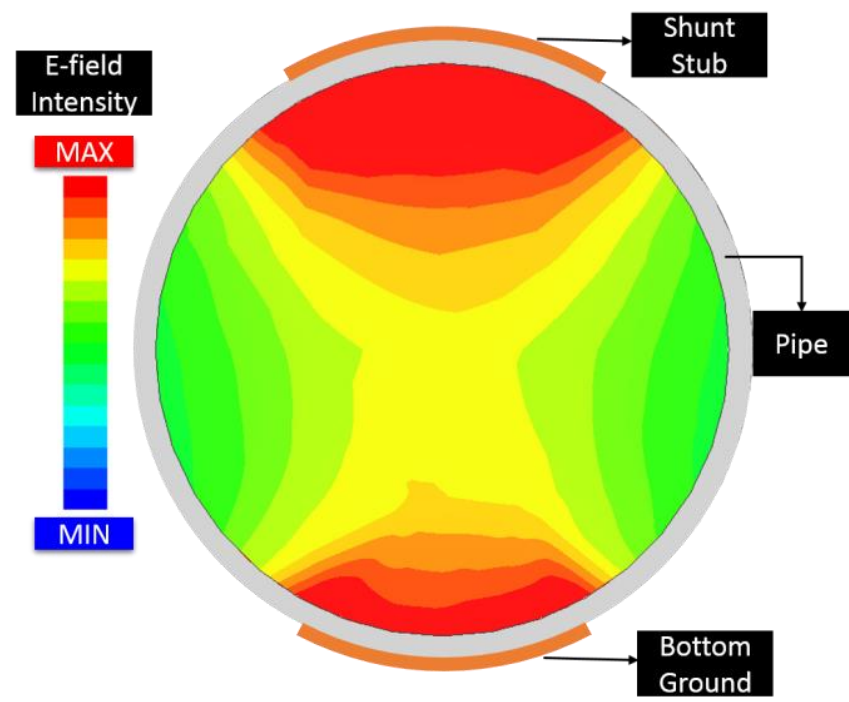

Fig. 2. Simulated E filed distribution inside the pipe in the case of flat implementation

The problem with this kind of E-filed distribution is that the resonant frequency of the T-resonator will be more sensitive to the substrate region between the signal and the ground conductor and less sensitive to the region on the side edges of the pipe. The non-uniformity of the E-fields inside the pipe makes the sensor (Fig. 1(b)) depend on the geometric distribution of oil and water in the pipe [15] which is not acceptable in real oil producing environment is which oil and water form complex distribution inside the pipe.

In order to quantify the dependence of the geometric distribution of the water and oil phase on the performance of the WC sensor, we consider oil and water as completely separate phases. The performance of the flat design has been simulated in HFSS for three test cases as shown in Fig. 3. In these test cases, the position of the top shunt stub and bottom ground plane have been kept the same while the oil and water phases have been rotated inside the pipe in a step of $90^{\circ}$.

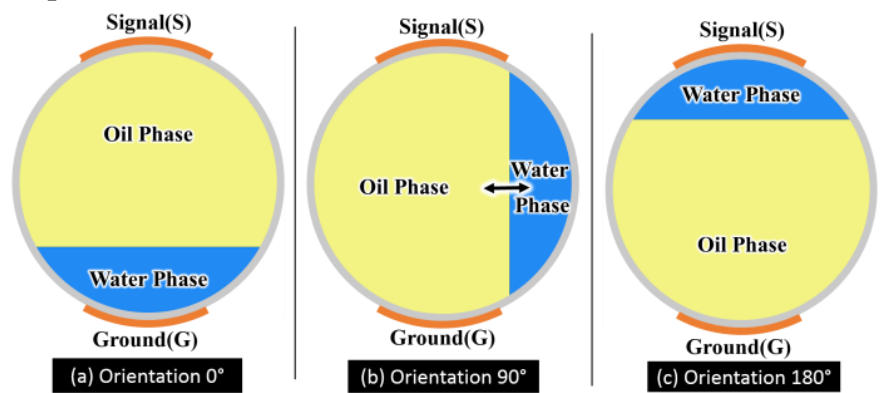

Fig. 3. Three test cases to quantify the dependence of orientation of the sensor with respect to the oil and water interface (a) $0^{\circ}($ reference $)(b) 90^{\circ}$ (c) $180^{\circ}$

It can be seen from Fig. 4 that the non-uniformity of the $\mathrm{E}$ field makes the sensor's response not only dependent on the WC but also on the geometric distribution of oil and water inside the pipe because $90^{\circ}$ orientation gives entirely different response than $0^{\circ}$ and $180^{\circ}$ response. The RMS error between the responses for $0^{\circ}$ and $90^{\circ}$ orientations was measured to be $28 \mathrm{MHz}$ which corresponds to an absolute error of around $35 \%$ in detecting the WC which is totallyunacceptable.

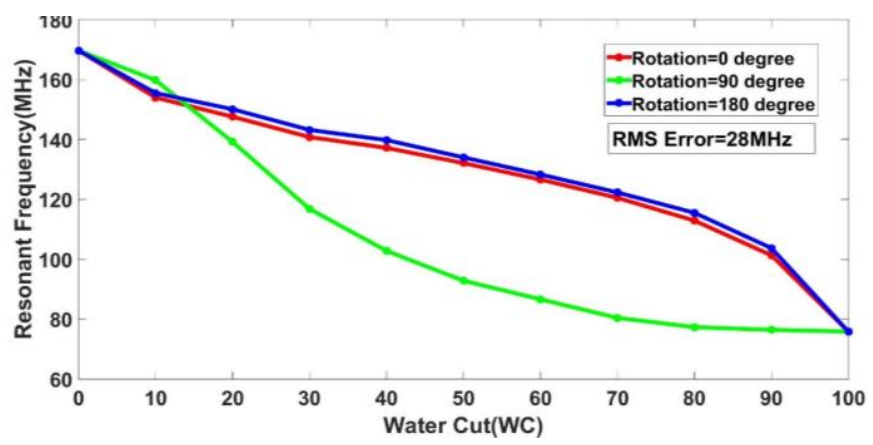

Fig. 4. Effect of different orientations on the performance of the flat design of the WC sensor

One easy solution for this problem (which most of the commercially available WC sensors adopt) is to break oil and water in so small particles that the particle size becomes less than $\lambda / 10$ at the operational frequency. The sensor can then treat oil and water as a single homogeneously mixed phase and does not depend on the geometric distribution any more. As highlighted in the introduction of this paper, such approach requires mixer or flow conditioners installed before the sensor which causes undesirable pressure drop in the pipelines. So, there is a need to come up with a new design which does not or minimally depend on the orientation (geometric distribution) of oil and water inside the pipe, without the need to mix them together.

\section{B. Orientation Insensitive Design (Helical Design)}

The $\mathrm{E}$ field distribution and simulated response of the flat design (Fig. 2 and Fig. 4) suggest that the WC sensor can become insensitive to orientation if the E-fields are distributed well inside the pipe.

Orientation insensitivity can be achieved by using a helical shaped signal and a ground conductor as shown in Fig. 5. In contrast to the flat design, the mutual rotation of the signal and ground conductors may help the E-fields (which exist between the signal and the ground conductor) to rotate inside the pipe such that the effective E field is distributed well inside the pipe. 


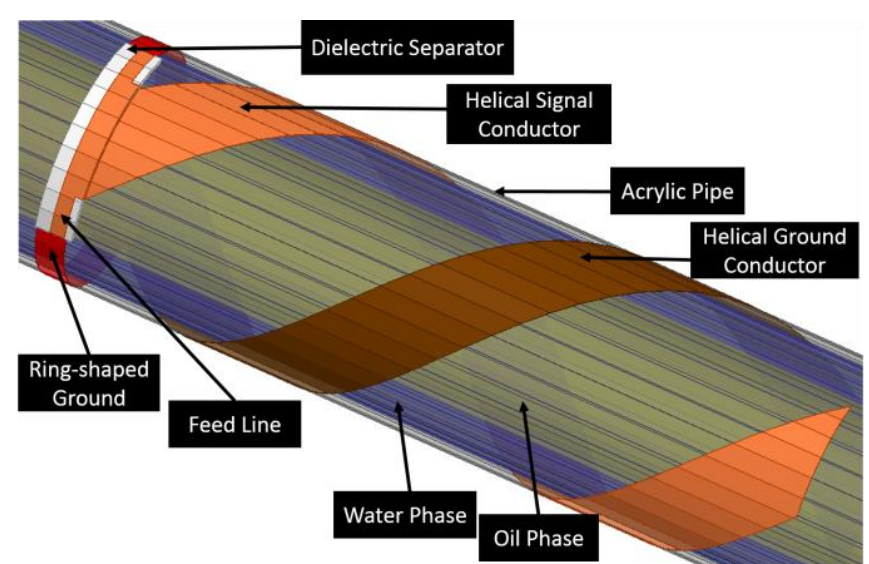

Fig. 5. Proposed helical shaped signal and ground conductors of T-resonator to achieve orientation insensitivity

In order to achieve minimum foot print of the sensor, the design shown in Fig. 5 has been optimized to have a single turn and the pitch equal to outer circumference ${ }^{2}$ of the pipe $(2 * \Pi * 25 \mathrm{~mm}=157 \mathrm{~mm})$. The reason for choosing this pitch value for the helical design is to keep the vertical and lateral distances between the signal and ground plane to be equal, i.e. $50 \mathrm{~mm}\left(25 \mathrm{~mm}^{* 2}\right)$. If the pitch is reduced, the signal and lateral ground ${ }^{3}$ will become closer than the signal and vertical ground ${ }^{4}$, which can reduce the sensitivity of the sensor. By increasing the pitch of the helix, fringing fields may reduce in which leads to higher sensitivity but at the expense of larger size.

The optimized design has been firstly simulated in HFSS for the radiation loss as the structure looks like a helical antenna. The realized gain turned out to be around $-39 \mathrm{~dB}$ at the resonant frequency, indicating that the structure is a bad radiator. After this, the homogeneity of the E-fields in the cross section of the pipe has been confirmed using the simulation results. The simulated transmission coefficient $\left(S_{21}\right)$ of the resonator has been plotted for the same three test cases (Fig. 3) which were considered for the flat design. The simulated response of the helical design for the three test cases is shown in Fig. 6. Comparing the response of the flat design (Fig. 4) with that of helical design (Fig. 6), it can be observed that the helical design is six times less sensitive to the geometric distribution of oil and water inside the pipe.

In order to explain the orientation insensitivity, we have plotted the E-fields of flat and helical versions of the resonator in Fig. $7 \mathrm{~s}$ at three equally spaced cross sections. The pipe has been intentionally hidden in Fig. 7 for the sake of clarity.

It is evident from Fig. 7, the E-field distribution inside the pipe remains the same over the length of the flat design (Fig. 7(a)) while the E field maxima rotates with the rotating signal and ground conductors for the case of the helical design (Fig. 7(b)). Effectively, the helical design makes the E fields distribute well inside the pipe. This is why the helical resonator is $6 x$ less sensitive to the geometric distribution of oil and water inside the pipe.

\footnotetext{
${ }^{2}$ The pipe used in simulations has outer radius of $25 \mathrm{~mm}$

${ }^{3}$ Part of helical ground lying on the same plane as signal

${ }^{4}$ Part of helical ground lying on other side of the pipe
}

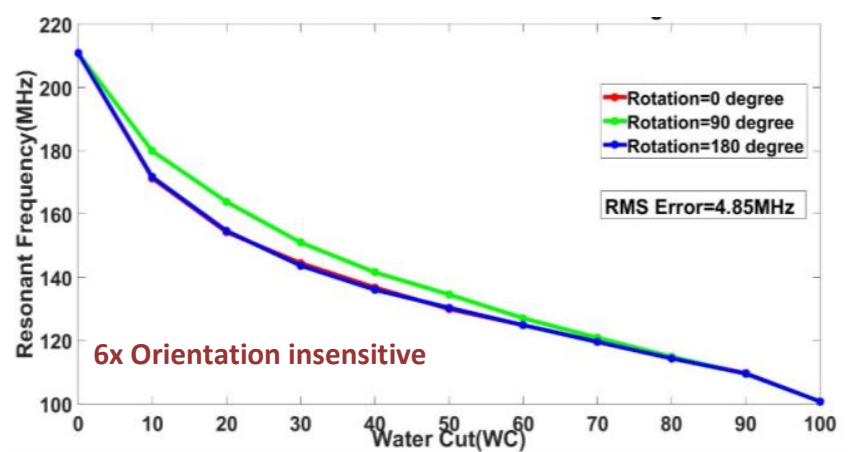

Fig. 6. Simulated response of the helical design of the WC sensor

Though the helical design of the T-resonator offers significant improvement over the flat design but $4.85 \mathrm{MHz}$ of the RMS error is still too large as it corresponds to an absolute error of $5 \%$ in detecting the WC.

\section{Improved orientation insensitive design}

Although the helical design has provided significant (6x) orientation insensitivity but the design is not $100 \%$ orientation insensitive yet. We can observe from Fig. 6 that the maximum discrepancy (although not as much as for the flat design-Fig. 4) still exists between the responses of $0^{\circ}$ and $90^{\circ}$ orientations. So, we are proposing to implement two mutually orthogonal helical resonators (with the same handedness ${ }^{5}$ ) on opposite sides of the ring ground. This way we can simultaneously obtain the response of the sensor at $0^{\circ}$ and $90^{\circ}$ which can then be averaged to get completely orientation insensitive response. Fig. 8 shows the HFSS simulation model of the ultimate design of the orientation insensitive WC sensor based on two helical stubs. =

\footnotetext{
${ }^{5}$ Handedness of the helix means whether the helix moves away or towards the observer by clockwise screwing motion with the line of sight in the helix's axis
} 


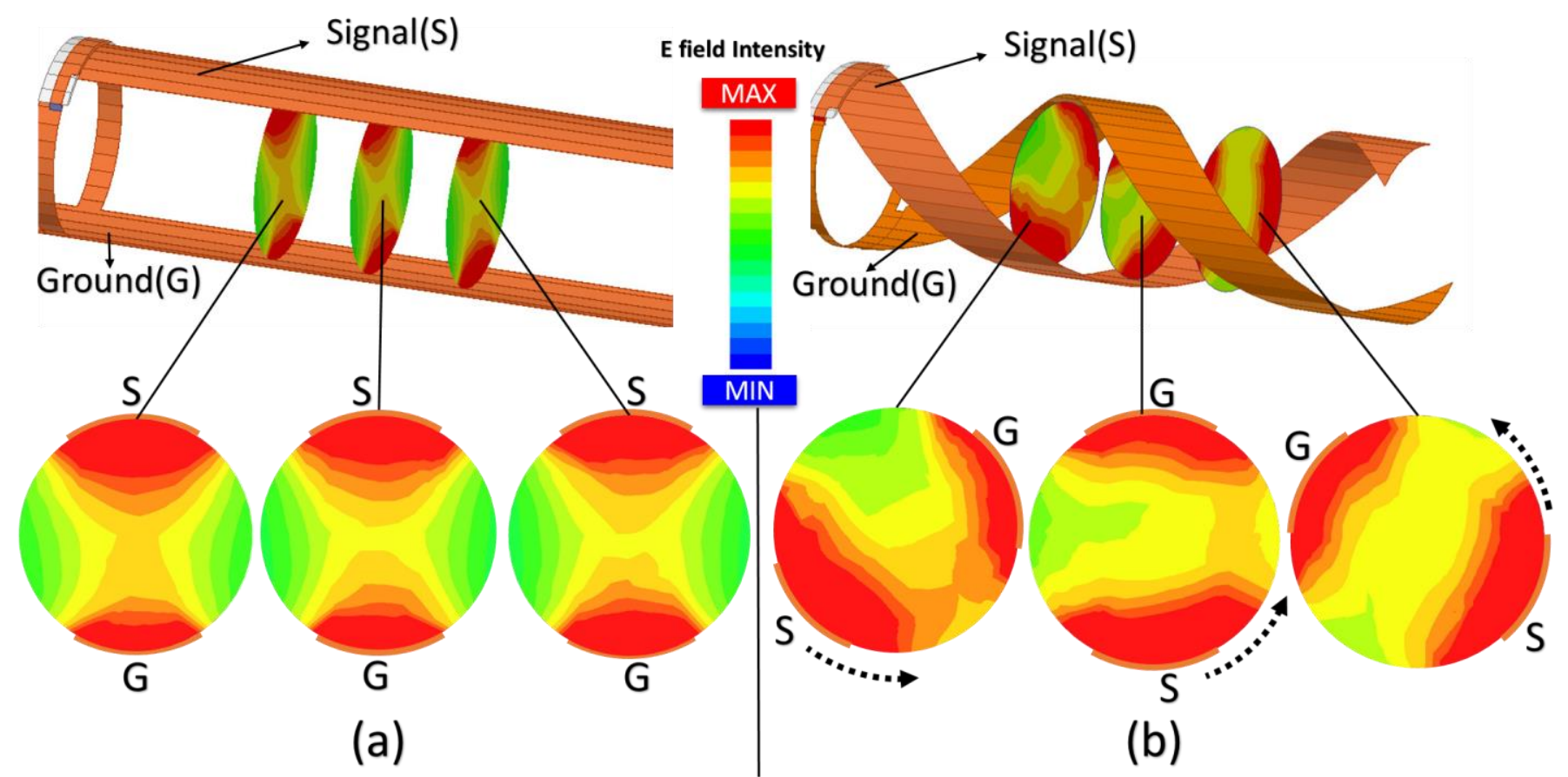

Fig. 7. E field distribution at three different equally spaced cross sections of the pipe (a) Flat design (b) Helical Design

Similar to the previous designs, the $S_{21}$ response for both the resonators has been simulated and their resonant frequencies have been averaged. In this simulation, oil and water phases have been rotated inside the pipe from $0^{\circ}$ to $180^{\circ}$ with a step of $30^{\circ}$. The averaged response for 7 different orientations is shown in Fig. 9. Comparing the response of the dual helical resonator based design (Fig. 9) with that of the flat design (Fig. 4), the new design can achieve 60 times more orientation insensitivity. The RMS error between $0^{\circ}$ and $90^{\circ}$ response is now reduced to only $0.48 \mathrm{MHz}$ which corresponds to an absolute error of just $0.5 \%$ which is 60 times better than the flat design.

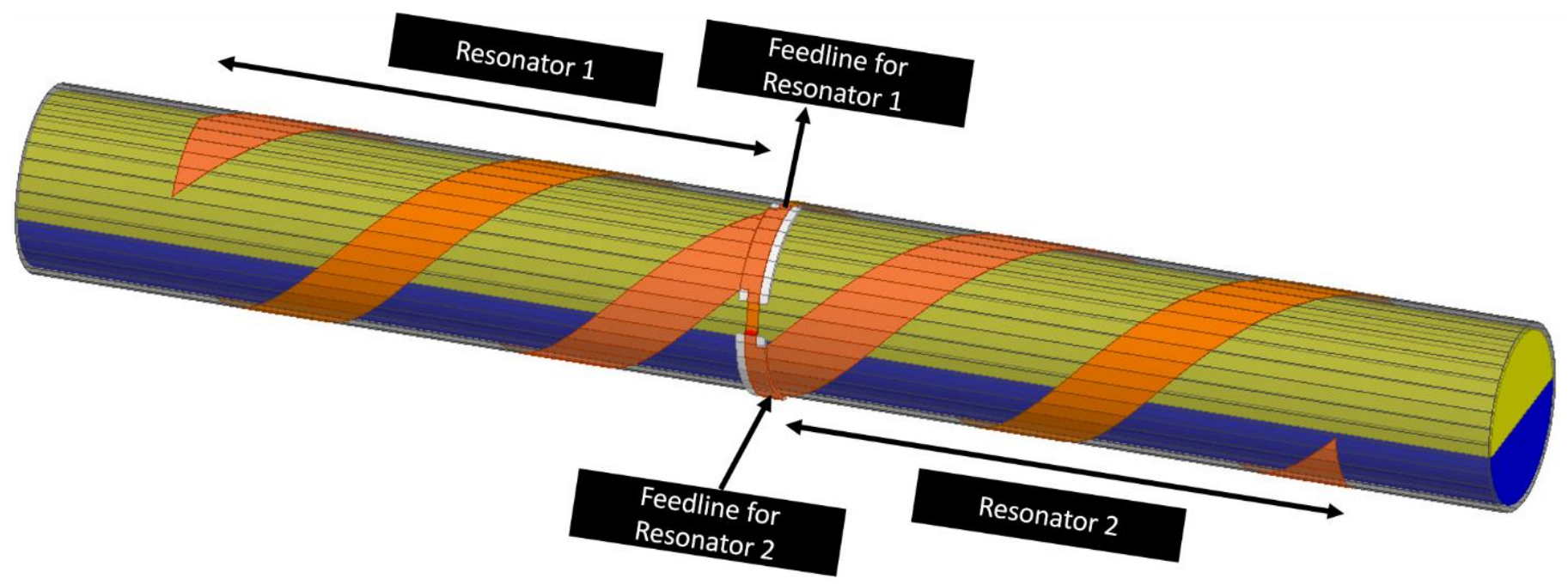

Fig. 8. Simulation design of dual helical stub based WC sensor 


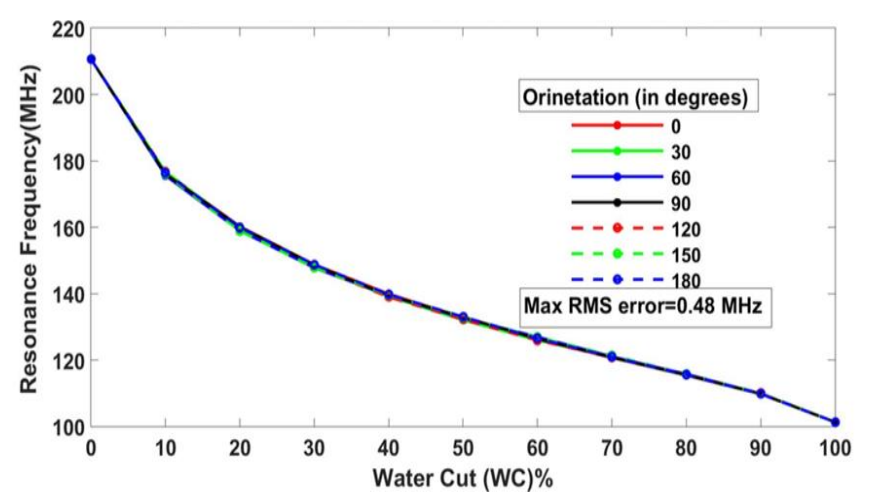

Fig. 9. Effect of orientation on dual helical resonator based WC sensor (60x orientation insensitive)

\section{FABRICATION}

After confirming that the WC sensor is no longer dependent upon the geometric distribution of oil and water inside the pipe, this design has been fabricated using silver paste and patterned using 3D printed mask. Step by step fabrication is shown in Fig. 10.

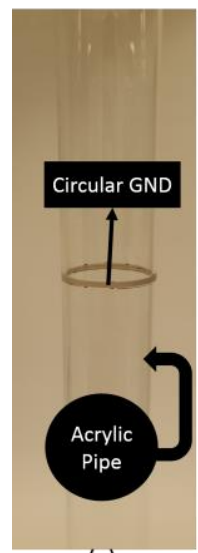

(a)

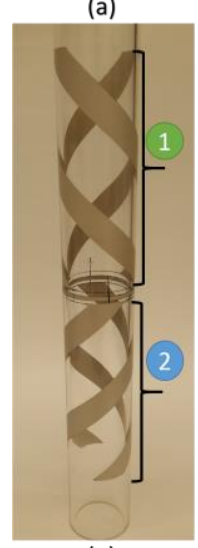

(e)
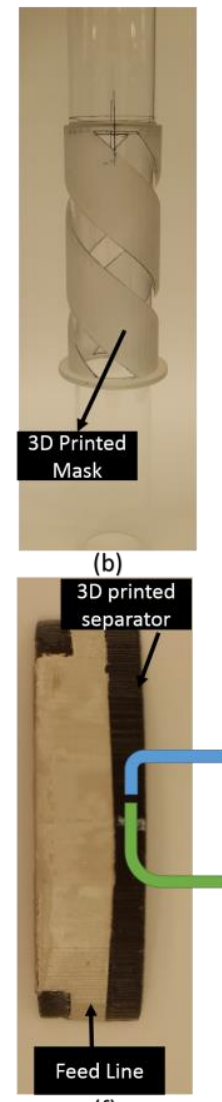

(f)

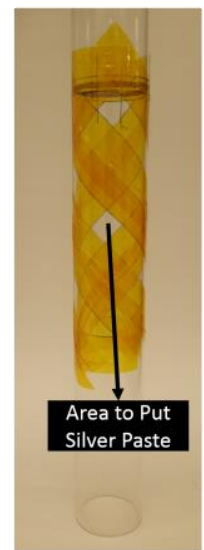

(c)

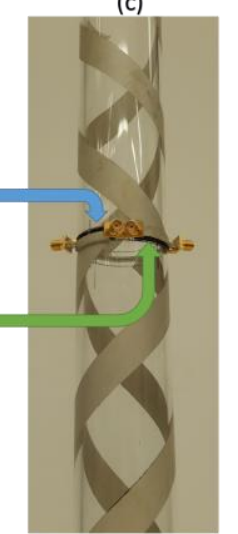

(g)
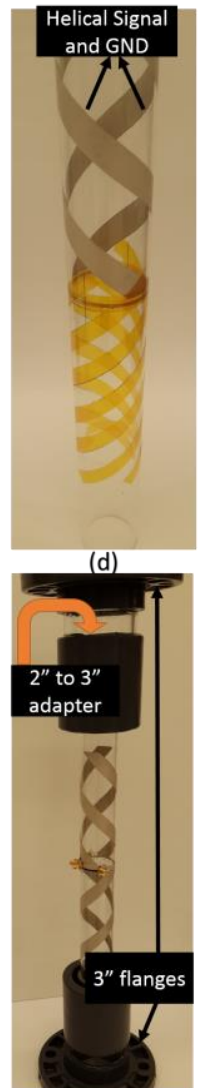

(h)

Fig. 10. Fabrication steps of dual-helical resonator based WC sensor

A circular shaped ring ground is patterned using silver paste on an acrylic pipe with outer diameter (OD) of $50 \mathrm{~mm}$ (Fig. 10(a)). In order to precisely pattern the helical resonators on top of the acrylic pipe, a 3D printed mask is fabricated using a 3D printer, i.e. Objet 260 connex 1 . This

mask has a hollow cylindrical interior with inner diameter (ID) of $50 \mathrm{~mm}$ so that it can be placed on top of the pipe surface. The mask has two hollow helical patterns one of which corresponds to the helical signal and the other to the helical ground (Fig. 10(b)). Markings have been made on the pipe surface with the mask placed on the pipe. The mask is then removed and the area which is not to be silver pasted, has been covered with the masking tape (Fig. 10(c)). LPKF pro-conduct silver paste was then poured on the pipe surface and leveled using squeegee. Masking tape is then removed and the similar procedure was repeated for the other resonator on the opposite side of the pipe (Fig. 10(d, e)). Similarly, feedline was also patterned on a 3D-printed dielectric separator piece (appearing as black in Fig. 10(f)) which was used to isolate the feedline from the ring ground. The feedline was then connected with the helical stub using conductive epoxy (Fig. 10(f, g)). The feedline on top of this separator piece helps launching a microwave signal from one port which is then received on the other port of the feedline after passing through the helical structure. SMA connectors are then mounted between the feedline and circular ground plane. Since the structure has two independent two port resonators, a total of four SMA connectors have been installed (Fig. 10(g)).

The flow loop in which the testing of the sensor had to be carried out, was based on 3" piping so that the diameter had to be converted from $2^{\prime \prime}(\sim 50 \mathrm{~mm})$ to $3^{\prime \prime}$. For this, $2^{\prime \prime}$ to $3^{\prime \prime}$ adapters along with $3^{\prime \prime}$ flanges were installed at each end of the prototype (Fig. 10(h)). As discussed in the first section of the paper, the flow regimes also depend on whether the sensor is installed horizontally or vertically. That is why, two identical prototypes were fabricated in order to simultaneously characterize the sensor in both the orientations.

\section{FLOW LOOP CHARACTERIZATION}

The fabricated prototypes have been characterized in the flow loop of Aramco Service Company, Houston, TX, which is shown in Fig. 11. Crystal Plus 70T mineral oil from STE Company was used in the flow loop whose dielectric properties were measured using Dielectric Assessment Kit (DAK-12) from SPEAG. The oil has a low loss with loss tangent below 0.001 and a dielectric constant of 2.1 in the desired operational frequency range (below 1 $\mathrm{GHz}$ ).

The storage tank of 500 gallons was filled up with the above mentioned oil and tap water. Dayton pumps in conjunction with electronically adjustable valves were used to control the flow rate of water and oil. Flow meters from Great Plains Industries were used to measure the flow rate of oil and water phases.

A LabVIEW GUI was built to control the valve positions and to view the real time flow rate of individual oil and water phases. All the control and feedback signals were being managed using a PLC by National Instruments. 


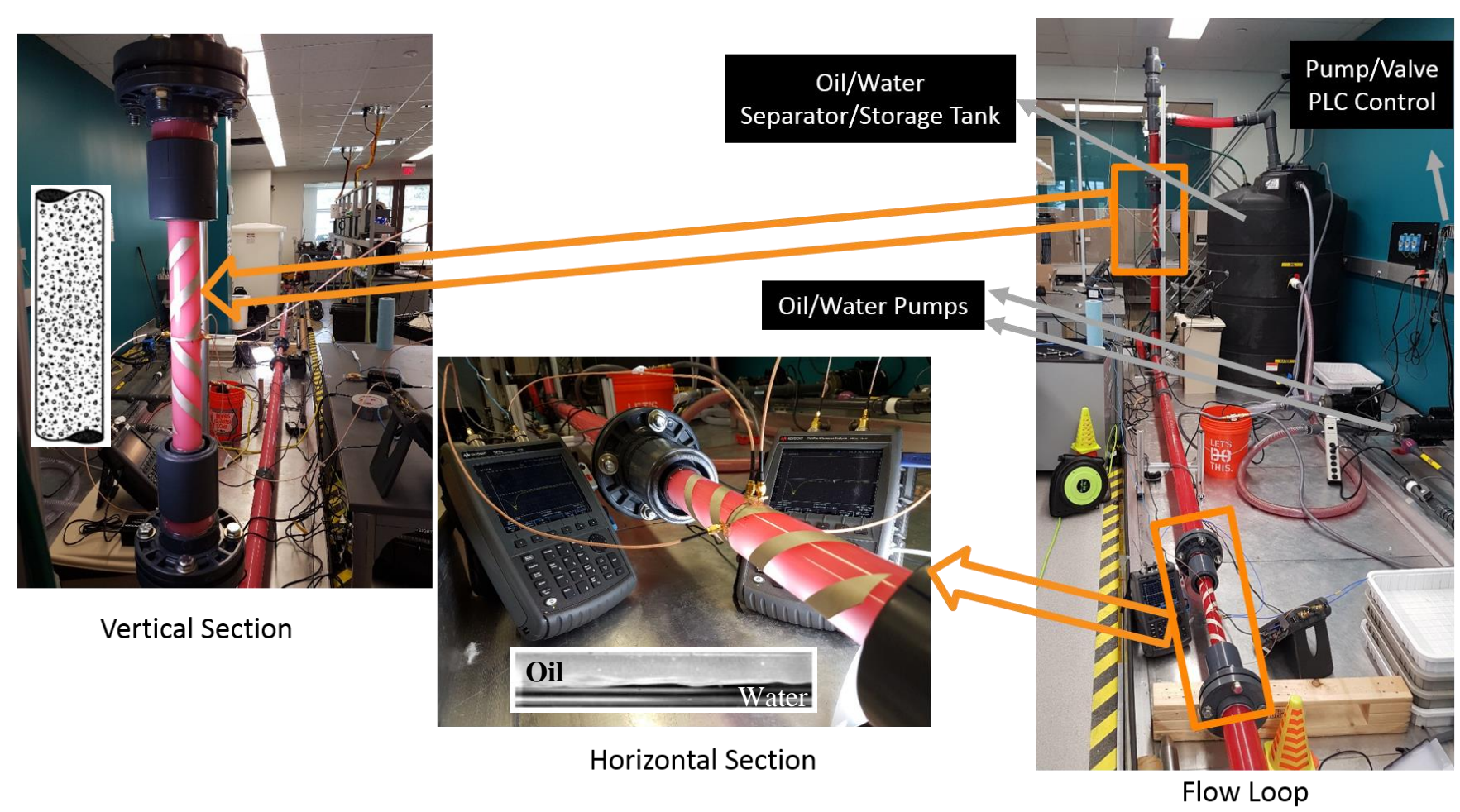

Fig. 11. Simultaneous horizontal and vertical characterization of the dual resonator based WC sensor in the industrial flow loop

It can be seen from the zoomed-in version of horizontal and vertical sections that the flow regimes are completely different at two different sections. The horizontal section experiences "wavy stratified" flow regime with less dense oil (appearing in red color) floating on top of heavy water (appearing as white color) [18]. The interface layer between oil and water becomes wavier by increasing the flow rate of oil and water phases. In contrast to the horizontal section, oil and water mixes up in the form of bubbles when they rise up in the vertical section resulting into "dispersed bubble" flow regime [18].

The sensor has been characterized in the vertical and horizontal positions simultaneously. For each sensor, the transmission coefficient $\left(\mathrm{S}_{21}\right)$ of two resonators are measured using handheld Agilent FieldFox VNA. The resonant frequency is extracted from the $S_{21}$ response, individually for two resonators and then averaged out to obtain a resonant frequency against each WC. This averaged resonant frequency is orientation insensitive and has been reported against the WC in all the characterization curves (Fig. 12 to Fig. 17) presented in this paper.

As stated earlier, the performance of the sensor does not get affected by the geometric distribution of oil/water phases in the pipe so, we characterized the sensor in 3 different orientations $\left(0^{\circ}\right.$ (reference), $45^{\circ}$ and $\left.90^{\circ}\right)$ to show orientation insensitivity. Moreover, the turbulence of the flow and the flow regimes also depend on the flow rate, the sensor has also been characterized for 3 different flow rates. Lastly, the sensor was also tested for the hysteresis effect, i.e. it was characterized by changing the $\mathrm{WC}$ in any direction (from high to low, then low to high WC) to see if the performance of the sensor depended on the history of the WC present in the pipe. The following sub-sections explain the characterization results.

\section{A. Orientation Effect}

The sensor was characterized at 3 different orientations $\left(0^{\circ}\right.$ (reference), $45^{\circ}$ and $\left.90^{\circ}\right)$ while keeping the flow rate at 50 gallons per minute (GPM) and changing the WC from $0 \%$ to $100 \%$ in all these cases. The averaged resonant frequency is plotted against each WC with a step size of 10\%. Fig. 12 and Fig. 13 show the characterized responses of WC sensor in vertical and horizontal orientations respectively.

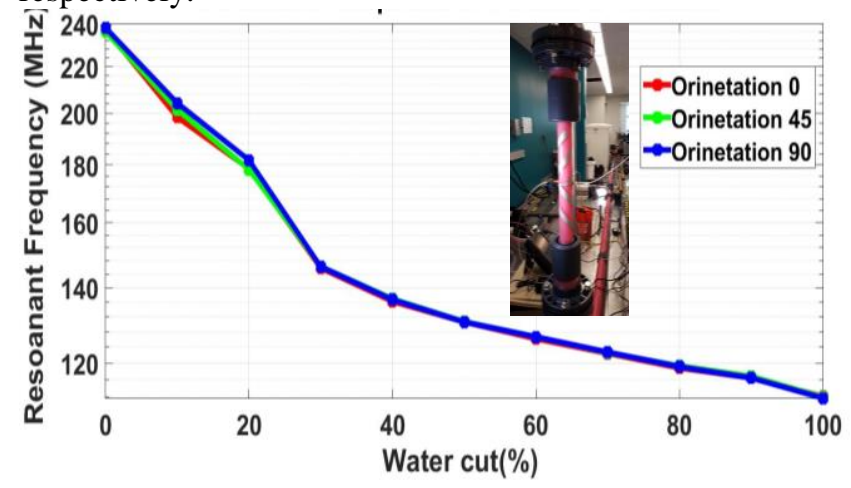

Fig. 12. Orientation effect on the performance of the WC sensor installed vertically (shown in inset)

Despite having completely different flow regimes, i.e. "dispersed bubble" and "wavy stratified" in vertical and horizontal sections, respectively, the sensor shows almost identical response as evident from Fig. 12 and Fig. 13. It makes this sensor especially suitable for use in modern lateral oil wells without requiring any preconditioner. 


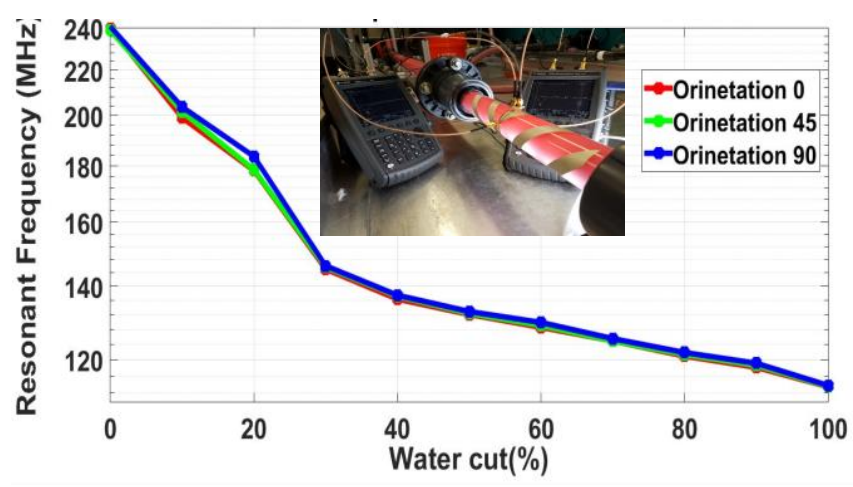

Fig. 13. Orientation effect on the performance of the WC sensor installed horizontally (shown in inset)

Since the E fields have been made to distribute well inside the pipe, different geometric distributions of oil and water in vertical and horizontal installations have no effect on the characteristic response of the sensor.

\section{B. Flow Rate Effect}

Reynolds number, given by (1), is a measure of turbulence of the flow. Where $\rho, \mathrm{v}$, and $\eta$ represent the density, relative speed and dynamic viscosity of the fluid, respectively, and $l$ is the characteristic length of the system.

$$
R e=\frac{\rho v l}{\eta}
$$

It is evident from the equation that the turbulence of the flow is directly proportional to the velocity of the fluid which may affect the geometric distribution of oil/water mixture inside the pipe. That is why, the sensors at vertical and horizontal positions were characterized at 3 different flow rates, i.e. 40 GPM, 45 GPM and 50 GPM, while keeping the orientation fixed at $0^{\circ}$ and changing the $\mathrm{WC}$ from $100 \%$ to $0 \%$ for all the three flow rates. Fig. 14 and Fig. 15 shows the characteristics response of the sensor at 3 different flow rates in vertical and horizontal positions, respectively.

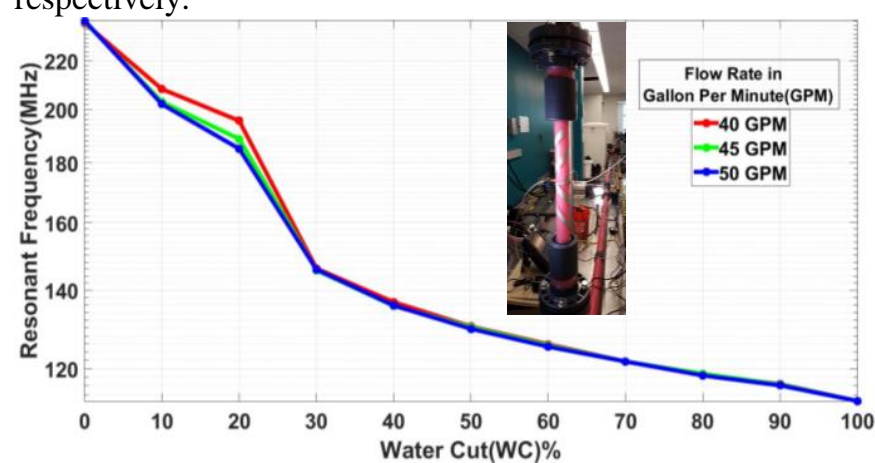

Fig. 14. Flow rate effect on the performance of the WC sensor installed vertically (shown in inset)

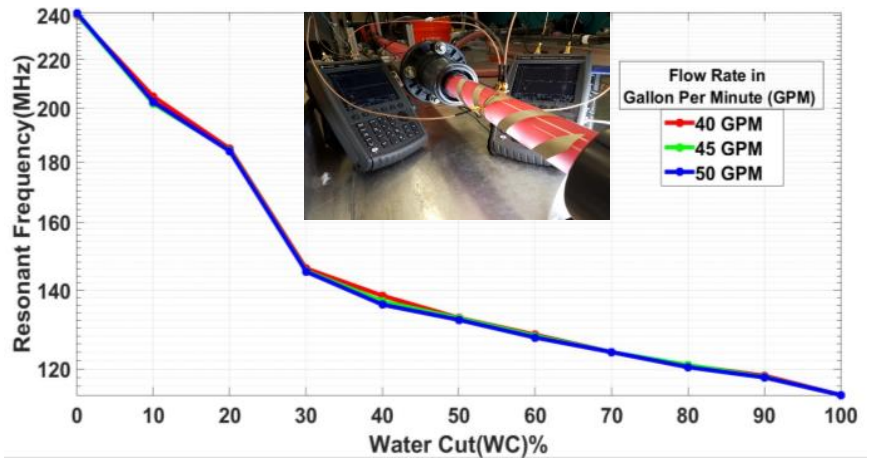

Fig. 15. Flow rate effect on the performance of the WC sensor installed horizontally (shown in inset)

Flow rate also does not affect the sensor performance significantly as evident from the overlapping response of the sensor at three different flow rates as shown in Fig. 14 and Fig. 15. A slight discrepancy was observed in the readings of $10 \%$ and $20 \% \mathrm{WC}$ at a flow rate of $40 \mathrm{GPM}$ flow rate that could have been caused by some human measurement error. Probably, the flow loop has not been run long enough to attain the designated WC. We may call this a pre-mature measurement error. Since the measurements were first taken on vertical prototype so we can observe this discrepancy in Fig. 14 but not in horizontal prototype (Fig. 15) which was measured afterwards. Oil might have attained its designated fraction in the flow loop by the time measurement was taken in horizontal section. Hysteresis Effect

The phase inversion is the point where oil and water interchange their role as the continuous or distributed phase inside a liquid-liquid multiphase flow. The phase inversion strongly affects the geometric distribution of oil and water inside the pipe and this point may vary depending on whether we move from oil continuous to water continuous (WC0 to $\mathrm{WC100)}$ or vice versa (WC100-WC0) [18]. In order to further confirm the independence of the sensor's response to geometric distribution, the sensor was characterized for full WC range in either direction, i.e. changing the WC from $0 \%$ to $100 \%$ and then from $100 \%$ to $0 \%$. This test was performed to confirm that the sensor's performance does not depend on the history of the WC in the pipe. Fig. 16 and Fig. 17 show the hysteresis effect on the WC sensor installed vertically and horizontally, respectively. 


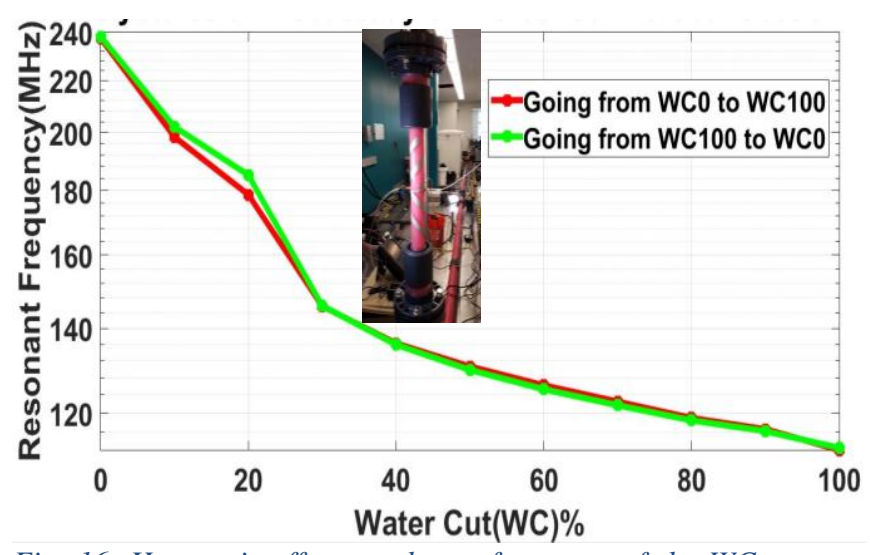

Fig. 16. Hysteresis effect on the performance of the WC sensor installed vertically (shown in inset)

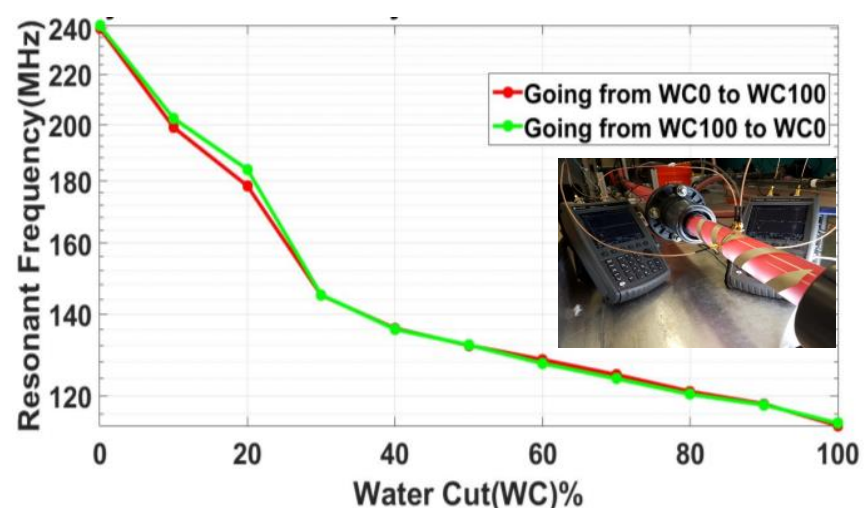

Fig. 17. Hysteresis effect on performance of the WC sensor installed horizontally (shown in inset)

Fig. 16 and Fig. 17 confirm that the sensor's response does not depend on the history of the WC present in the pipe rather it depends on the absolute value of the WC which is desirable.

\section{PERformance Test}

After characterizing the designed WC sensor in the flow loop, a custom MATLAB GUI (Fig. 19) was developed to check its performance in random test conditions. Two identical prototypes were installed in random orientation at horizontal and vertical test sections and random WCs were established at random flow rates in the flow loop. MATLAB GUI handles the user interaction for acquiring the $S_{21}$ data from four VNAs (two for each prototype) by running Standard Commands for Programmable Instruments (SCPI) on its backend. System block diagram is shown in Fig. 18.

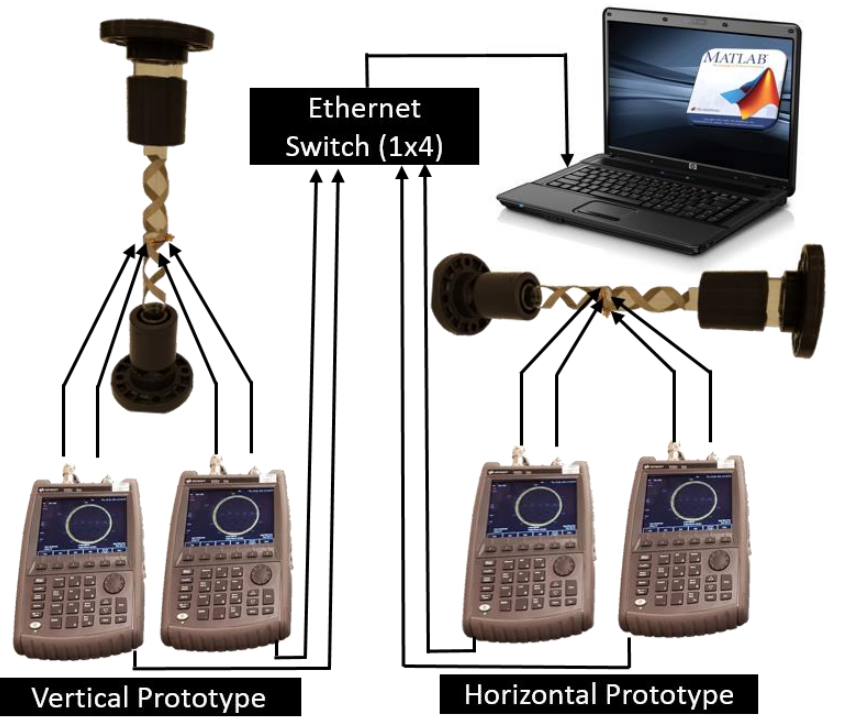

Fig. 18. Block diagram of simultaneous testing of horizontal and vertical prototypes using MATLAB GUI

After acquiring the $S_{21}$ data from all VNAs, MATLAB code extracts the resonant frequency and compares it with the hard-coded characterization curves of the sensor already fed into the system. Look-up table based correspondence translates the resonant frequency into the WC.

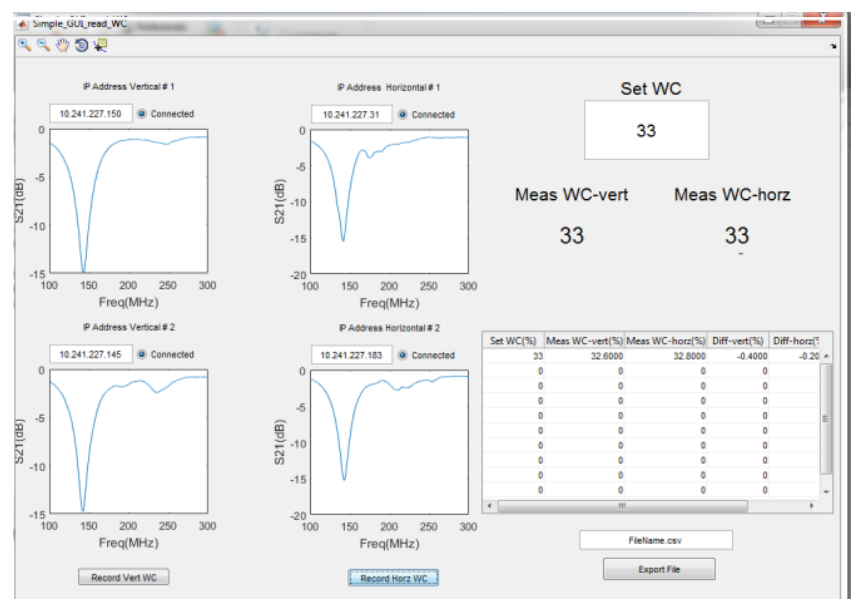

Fig. 19. Screenshot of the custom MATLAB GUI dealing with data acquisition, post processing and translation of resonant frequencies to the water cut (WC)

The performance of horizontally as well as vertically installed sensors was simultaneously tested at multiple random points over full $\mathrm{WC}$ range. 


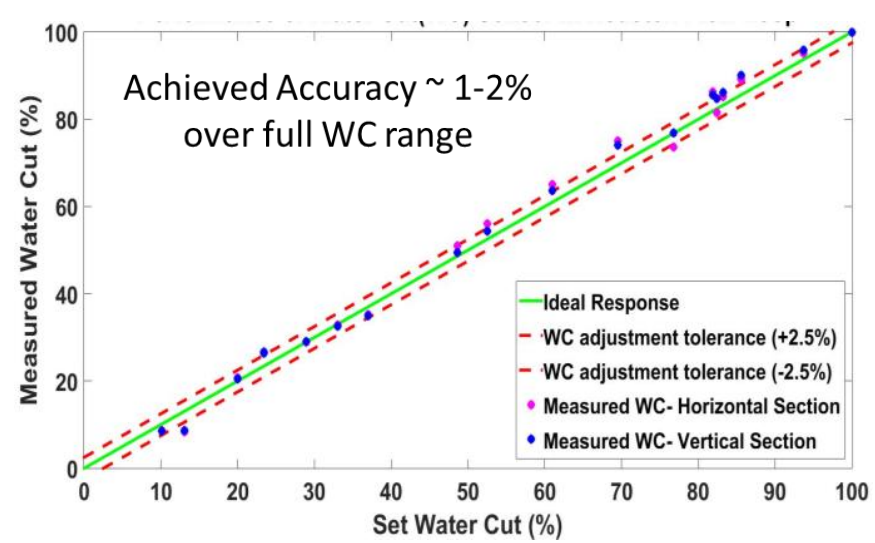

Fig. 20. Performance of the WC sensor (Both in vertical and horizontal installation) in the flow loop

It's worth mentioning here that the $\mathrm{WC}$ in the flow loop can only be set with a tolerance of $\pm 2.5 \%$. The green line corresponds to the ideal response with $0 \%$ error while red dotted curves represents $\pm 2.5 \%$ tolerance in adjusting the WC in the flow loop. Blue and pink dots show the measured WC in vertical and horizontal orientation, respectively, against a particular set WC. It is evident that almost all the measured points are within the tolerance limits of the flow loop. Taking the uncertainty of the flow loop into account, the WC sensor shows an accuracy of 1$2 \%$ over the full WC range in both the vertical and horizontal installations. Moreover, it can easily be observed that vertical and horizontal sections measure the same WC disregarding a huge difference in the flow regimes existing in the corresponding sections.

\section{CONCLUSION}

This paper has successfully shown the design, low cost fabrication and industrial flow loop testing of a microwave based WC sensor. Due to the mutually orthogonal dual helical open stub based design, E-fields have been distributed well inside the pipe. Resultantly the sensor is sensitive only to volumetric fraction of water in oil regardless of its orientation, flow rate and geometric distribution of oil and water inside the pipe. Unlike many commercial water-cut sensors, our sensor's response remains identical in vertical as well as in horizontal installations without requiring any preconditioner which may cause undesirable pressure drop in the pipe lines. The full WC range accuracy of 1-2\% has been achieved despite the limitations of the test setup. One may integrate an oscillator with the resonators in order to eliminate the need of VNA to record its resonant frequency and to develop an extremely low cost self-contained water-cut sensor in the future.

\section{REFERENCES}

[1] D. J. Xiao, "Determining Water Volume Fraction for Oil-Water Production with Speed of Sound Measurement," Saudi Aramco Journal of Technology, 2015.

[2] D. o. T. a. I. (UK), "Guidance Notes for Petroleum
Measurement," Oil and Gas Authority, 2003.

[3] EESIFLO, "watercut-meter," 2012. [Online]. Available: http://www.eesiflo.com/watercutmeter.pdf.

[4] G. Lucas, "Towards a phase-distribution-independent impedance volume-fraction measurement," Flow Measurement and Instrumentation, vol. 2, no. 2, pp. 105-114, 1991.

[5] J. T. K. O. E.A. Hammer, "Capacitance transducers for non-intrusive measurement of water in crude oil," Flow Measurement and Instrumentation, vol. 1, no. 1, pp. 51-58, 1989.

[6] V. F. D. S. P. P. Marco Demoria, "A capacitive sensor system for the analysis of two-phase flows of oil and conductive water," Sensors and Actuators A: Physical, vol. 163, no. 1, pp. 172-179, 2010.

[7] P. Dynamics, "Installation and Instruction ManualFull Range Water in Hydrocarbon Analyzer," [Online]. Available: http://www.phasedynamics.com/images/Analyzerdoc uments/0063-00003-000RevB.pdf.

[8] A. P. L. A. S. S. Yu. V. Makeev, "Microwave measurement of water content in flowing crude oil," Automation and Remote Control, vol. 74, no. 1, pp. 157-169, 2013.

[9] R. A. Hatinoor, The effect of gas on microwave resonance frequency meter technology- Masters Thesis, University of Stavanger - Norway, 2013.

[10] T. H. M. Reza Taherian, "MICROWAVE DEVICE AND METHOD FOR MEASURING MULTIPHASE FLOWS". Patent 5,485,743, 23 jan 1996.

[11] S. Ashton, "Development And Trial Of Microwave Techniques For Measurement Of Multiphase Flow Of Oil, Water And Gas," in SPE Asia Pacific Oil and Gas Conference, Melbourne, 1994.

[12] Weatherford, "Red Eye Series of Water-Cut Meters," 2015. [Online]. Available: http://www.weatherford.com/doc/wft297766.

[13] Schlumberger, "Vx Spectra Surface Multiphase Flowmeter," [Online]. Available: http://www.slb.com/services/characterization/testing/ multiphase/spectra.aspx.

[14] H. K. T. Frøysteina, "Dual energy gamma tomography system for high pressure multiphase flow," Flow Measurement and Instrumentation, vol. 16, no. 2-3, pp. 99-112, 2005.

[15] M. A. A. S. Muhammad Akram Karimi, "Low cost and pipe conformable microwave-based water-cut sensor," IEEE Sensors Journal, no. 99, 2016.

[16] M. K. J.-P. S. a. P. S. K.-P. Lätti, "A Review of Microstrip T-Resonator Method in Determining the Dielectric Properties of Printed Circuit Board Materials," IEEE TRANSACTIONS ON INSTRUMENTATION AND MEASUREMENT, 2007.

[17] G. McKerricher, D. Conchouso, B. S. Cook, I. Foulds 
and A. Shamim, "Crude oil water-cut sensing with disposable laser ablated and inkjet printed RF microfluidics," in IEEE MTT-S International

Microwave Symposium (IMS2014), Tampa, 2014.

[18] K. H. Ngan, "Phase Inversion in dispersed liquidliquid pipe flow," University College London, London, 2010.

[19] DELTA-C, "Water in oil monitor, insertion systemDC-3510," [Online]. Available:

http://www.ima.co.uk/products/by-

application/refined-oil-quality-instruments/water-inoil-measurement-dc-3510f.

[20] "A Microwave Instrument for the Continuous Monitoring of the Water Content of Crude Oil," Proceedings of the IEEE, vol. 62, no. 1, pp. 103-108, 1974. 